\title{
The vitamin $D$ receptor and the etiology of RANTES/CCL-expressive fatty-degenerative osteolysis of the jawbone: an interface between osteoimmunology and bone metabolism
}

This article was published in the following Dove Press journal:

International Journal of General Medicine

\author{
Johann Lechner' \\ Jürgen Aschoff ${ }^{2}$ \\ Tatjana Rudi ${ }^{3}$ \\ 'Clinic for Integrative Dentistry, \\ Munich, Germany; ${ }^{2}$ Center for \\ Integrative Healing, Wuppertal, \\ Germany; ${ }^{3}$ Statistics at Institute \\ for Epidemiological Studies, Berlin, \\ Germany
}

Background: Recent research on vitamin D indicates that our current understanding of the factors leading to chronic inflammation should be revised. One of the key mechanisms by which microbial immunosuppression occurs is the suppression of one of the most common endogenous cell nucleus receptors: the vitamin D receptor (VDR). Autoimmune diseases may be correlated with VDR deactivation (VDR-deac) which occurs when the receptor is no longer able to transcribe antimicrobial agents. Excess 1,25-dihydroxyvitamin D $(1,25 \mathrm{D})$ is not converted to 25-hydroxyvitamin D (25D); thus, high 1,25D levels may be accompanied by low 25D values.

Patients and methods: Since $1,25 \mathrm{D}$ promotes osteoclast activity and may thereby cause osteoporosis, fatty-degenerative osteolysis of the jaw (FDOJ), as described by our team, may also be associated with VDR-deac. In 43 patients, vitamin D conversion, immune system function and the quality of bone resorption and formation in the jawbone were related factors that may enhance chronic inflammatory processes. Here, we examine the relationship between immunology and bone metabolism among 43 FDOJ patients and those with immune system diseases (ISDs). Results: We provide a link between FDOJ, RANTES/CCL5 overexpression and VDR-deac. Conclusion: The clinical data demonstrate the interaction between VDR-deac and proinflammatory RANTES/CCL5 overexpression in FDOJ patients.

Keywords: vitamin D receptor; chemokine RANTES/CCL5, osteopathy of the jawbone, immune system diseases, osteoimmunology

\section{Introduction}

The etiology of chronic inflammatory diseases, such as rheumatoid arthritis, multiple sclerosis, systemic lupus erythematosus and arteriosclerosis, has not been elucidated. It is widely acknowledged that several factors are linked to the development of such diseases, including genetic predisposition and environmental and dietary factors. Recently, modern metagenomic research on intra- and extracellular genes has shown that over the course of centuries, thousands of microbes have succeeded in establishing themselves permanently in our bodies. A clever mechanism directed to this end is the deactivation of the vitamin D receptor (VDR); intraphagocytic bacteria produce ligands that deactivate the VDR. In turn, pathogenic bacteria have developed mechanisms to alter and evade the host immune response. ${ }^{1,2}$ In this way, interleukin (IL)-2 and interferon gamma (IFN- $\gamma$ ) switch off the body's own innate immunity which makes possible an immunogenic reaction, particularly in the case of intracellular microorganisms. ${ }^{3}$ Researchers have found that autoimmune disease
Correspondence: Johann Lechne Gruenwalder Str. I0A, 8I547 Munich, Germany

Tel +49896970129

Email drlechner@aol.com 
markers can be greatly increased in the presence of chronic mycobacterial infections. ${ }^{4}$ Advances in detection techniques using improved genome-based cultivation methods are highly likely to significantly expand the number of known pathogens involved in chronic diseases. ${ }^{5-7}$ It is increasingly recognized that bacteria can persist as cell wall-deficient variants (otherwise known as L-forms) ${ }^{5-7}$ and as "persistent" forms within metagenomic bacterial communities. ${ }^{8,9}$ By means of the VDR, 1,25-dihydroxyvitamin D3 $\left(1,25[\mathrm{OH}]_{2} \mathrm{D}_{3} / 1,25 \mathrm{D}\right)$ regulates the immune system which is present in most immune cell types, particularly monocytes, macrophages and dendritic cells. ${ }^{10}$ In general, the innate immune system is enhanced and the adaptive immune system is inhibited by $1,25 \mathrm{D} .{ }^{11,12}$ Thus, an effective immune response is heavily dependent on the vitamin $\mathrm{D}$ endocrine system which is responsible for balancing inflammatory and anti-inflammatory processes. ${ }^{13}$ The VDR transcribes over 1,400 genes which accounts for $4 \%$ of all human genes and which are no longer able to be transcribed when the VDR is deactivated. The VDR also affects parathormone and calcium-sensitive receptors and is thus essential for maintaining well-regulated calcium homeostasis in the bone. Hence, the VDR is further implicated in systemic disorders of calcium metabolism, as well as vitamin D utilization in fatty-degenerative osteolysis of the jawbone (FDOJ). ${ }^{14}$

\section{Research aims}

The purpose of this article was to investigate the extent to which deactivated VDR (VDR-deac) plays an etiological role in the development of chronically altered metabolism in the jawbone in a cohort of patients with immune system diseases (ISDs). This article also attempts to provide further insight with respect to the question of whether VDR-deac and inflammatory cytokine overexpression in FDOJ areas may function as a reinforcement loop in ISDs.

\section{Materials and methods}

The study presented herein was performed as a casecontrol study and was deemed to be retrospective in nature. Approval was granted by Institute for Medical Diagnostics (IMD)-Berlin forensic accredited Institute DIN EN 15189/ DIN EN 17025. All patients provided their written consent to participate in this study, and the samples and data were collected in the course of routine practice, i.e., regular oral surgery procedures. The study cohort of 43 patients with FDOJ comprised of five groups consisting of patients with various specialist-diagnosed ISDs: atypical facial and trigeminal pain $(n=9)$; neurodegenerative disorders (multiple sclerosis and amyotrophic lateral sclerosis) $(n=5)$; tumors (breast cancer and prostate cancer) $(n=5)$; rheumatism (fibromyalgia and Lyme disease) $(n=16)$; and chronic fatigue syndrome $(n=8)$. The patients' average age was 54.05 years (age range: $23-75$ years). There was a gender ratio (females to males) of 25:18. A control group comprised of 19 patients without FDOJ, had an average age of 54 years (age range: 38-71 years) and a gender ratio (female to male) of $8: 11$. All patients were seeking to uncover the etiology of their respective ISDs, including possibly FDOJ-induced "silent inflammation" of the jawbone. The serum values of 25-hydroxyvitamin D3 $(25[\mathrm{OH}]$ $\mathrm{D}_{3} / 25 \mathrm{D}$ ) and $1,25 \mathrm{D}$ were determined for the cohort. Following the clinically necessary excision of FDOJ, conspicuous FDOJ samples were analyzed for their cytokine content. Serum vitamin D levels were analyzed in FDOJ group $(n=43)$. Cytokine levels in the jawbone were analyzed in FDOJ group $(n=43)$ and the control group $(n=19)$.

The use of medications for the treatment of systemic diseases was not generally regarded as an exclusion criterion. Alcohol addiction, fetal alcohol syndrome or abuse of any other drugs were additional exclusion criteria. ${ }^{15,16}$ Defined exclusion criteria consisted of cortisone and bisphosphonate use due to their effects on bone metabolism as well as current or recent use of vitamin D preparations, i.e., within the previous 14 days. The patients' serum vitamin D levels were correlated with the cytokine profiles of FDOJ areas of the five patient groups.

\section{Serum measurement of 25D and I,25D}

The analysis of $1,25 \mathrm{D}$ was carried out by means of the chemiluminescence immunoassay IDS-iSYS 1,25 VitD ${ }^{X p}$ with the analytical device iSYS (International Decision Systems, Minneapolis, MN, USA). The analysis of 25D levels was carried out using the chemiluminescence immunoassay LIAISON ${ }^{\circledR} 25 \mathrm{OH}$ Vitamin D TOTAL with the LIAISON ${ }^{\circledR}$ analyzer (DiaSorin, Saluggia, Italy).

\section{Cytokine profile measurement in jawbone osteolysis}

The current treatment for FDOJ lesions consists of bony cavity curettage. ${ }^{17,18}$ To elucidate a possible causative link between FDOJ and VDR-deac at the Munich Clinic for Integrative Dentistry (Munich, Germany), 43 patients with ISDs who were also diagnosed with FDOJ underwent surgery on the affected jaw area. Following the administration of local 
anesthesia, the mucoperiosteal flap was folded over and the cortical layer was removed. All patients exhibited FDOJ within the bone marrow that was similar to FDOJ samples described in the literature. ${ }^{19,20}$ In all 43 cases, surgery was performed on edentulous jaw areas at the sites of former wisdom teeth and the adjacent retromolar areas.

In 62 jawbone samples (43 FDOJ samples from the ISD group and 19 healthy jawbone samples), seven mediating factors were measured: fibroblast growth factor (FGF)-2, IL-1 receptor antagonist (IL-1ra), IL-6, IL-8, monocyte chemotactic protein (MCP)-1, tumor necrosis factor alpha (TNF- $\alpha$ ) and RANTES/CCL5 (R/C). The FDOJ clumps with a volume of up to $1 / 2 \mathrm{~cm}^{3}$ were scooped out (Figure 1 shows a clinical example of their morphology), and these pea-sized clumps of tissue were immediately placed in a sterile container (Sarstedt micro-tube; ref. 72.692.005) which was sealed and stored at $-20^{\circ} \mathrm{C}$ until transportation to the laboratory (IMD-Berlin, Berlin, Germany). There, the tissue samples were mechanically comminuted, incorporated with $200 \mu \mathrm{L}$ of protease buffer (Complete Mini Protease Inhibitor Cocktail; Hoffmann-La Roche, Basel, Switzerland) and homogenized. The homogenate was centrifuged for $15 \mathrm{~min}-$ utes at 13,400 rpm, after which the supernatant was removed and centrifuged for another 25 minutes at 13,400 rpm. The determination of $\mathrm{R} / \mathrm{C}$ was carried out in the supernatant of the tissue homogenate using the Human Cytokine/Chemokine Panel I (MPXHCYTO-60K; Millipore GmbH, Schwalbach, Germany), according to the manufacturer's protocol and using the Luminex $200^{\mathrm{TM}}$ with xPonent ${ }^{\circledR}$ software (Luminex, Austin, TX, USA).

\section{Statistical analysis}

The measured values obtained from the FDOJ and control cohorts were subjected to descriptive statistical analyses using SAS 9.4. The median, mean and distribution of the data

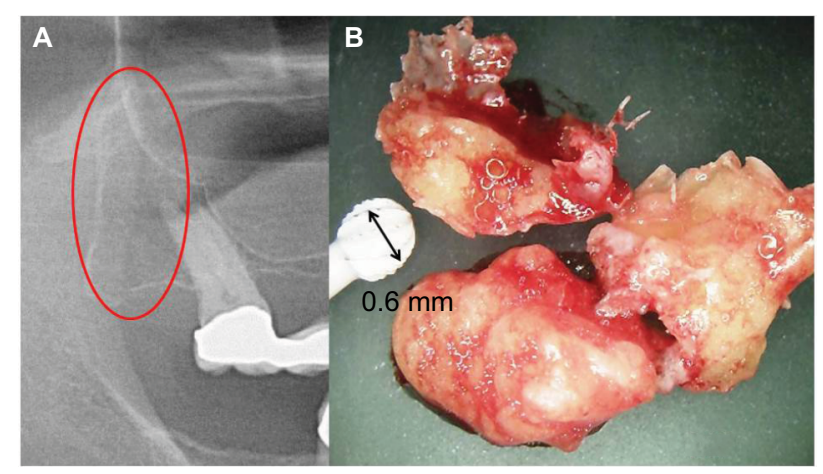

Figure I (A) Red oval shows location of fatty-degenerative osteolysis of the jawbone; (B) 1:15 scale, morphology of fatty-degenerative osteolysis. were determined to assess whether nonparametric or parametric tests were most appropriate. The difference between the values of FDOJ patients and the general population was assessed using Student's $t$-test. Differences between the two cohorts were determined using the Wilcoxon-Mann-Whitney test. The differences between disease groups were tested with the Kruskal-Wallis test. Statistical significance was set at a $P<0.05$.

\section{Results \\ Distribution of I,25D and 25D in the total cohort}

For male and female adults, the elderly, and both summer and winter values, a mean level of $22-111 \mu \mathrm{g} / \mathrm{L}$ was assumed for cholecalciferol 25D. The mean level of calcitriol 1,25D was assumed to be 18-64 ng/L for the same distribution (standard values were obtained from German Laborwerte Verzeichnis). In the recent literature, ${ }^{3,21}$ values $>45$ are considered as suspicious, and for this reason the upper reference value was set to $50 \mathrm{ng} / \mathrm{L}$ in our statistical analyses. As is well known, only one-thousandth of the amount of $1,25 \mathrm{D}$ is present in the blood when compared to $25 \mathrm{D}$. The distribution of these values in the total study cohort $(n=43)$ is shown in Figure 2.

\section{The ratio of 25-hydroxy-(cholecalciferol) and I,25-dihydroxy-(calcitriol) vitamin D3 in determining the deactivation of the vitamin $D$ receptor}

We calculated the ratio of $1,25 \mathrm{D}$ (measured in $\mathrm{ng} / \mathrm{L}$ ) and $25 \mathrm{D}$ (measured in $\mu \mathrm{g} / \mathrm{L}$ ) in 43 patients with systemic immunological disorders (Figure 2), and clinically verified the presence of FDOJ according to recommendations presented in the literature. ${ }^{21}$ This may pose a problem, as there is a thousandfold difference in the concentrations of the two vitamins in question. The direct detection of VDR-deac is not yet possible in standard laboratories, however, since it is a cell nucleus receptor that is found in the mitochondria. In practice, a simple reliable measurement is necessary to determine the status of the complex system of vitamin D regulation. It is possible to infer from the discussion in the relevant literature that a ratio $>1.3$ may be considered as a reference value for VDRdeac. ${ }^{22-24}$ The hypothesis that the mean value of the ratios in the FDOJ group is $\leq 1.3$ may be rejected $(P<0.001)$. Furthermore, the descriptive analysis indicates that 34 patients with ISDs from the FDOJ study cohort ( $\mathrm{n}=43$ ) showed VDR-deac. As Figure 3 illustrates, in all five disease groups the mean 


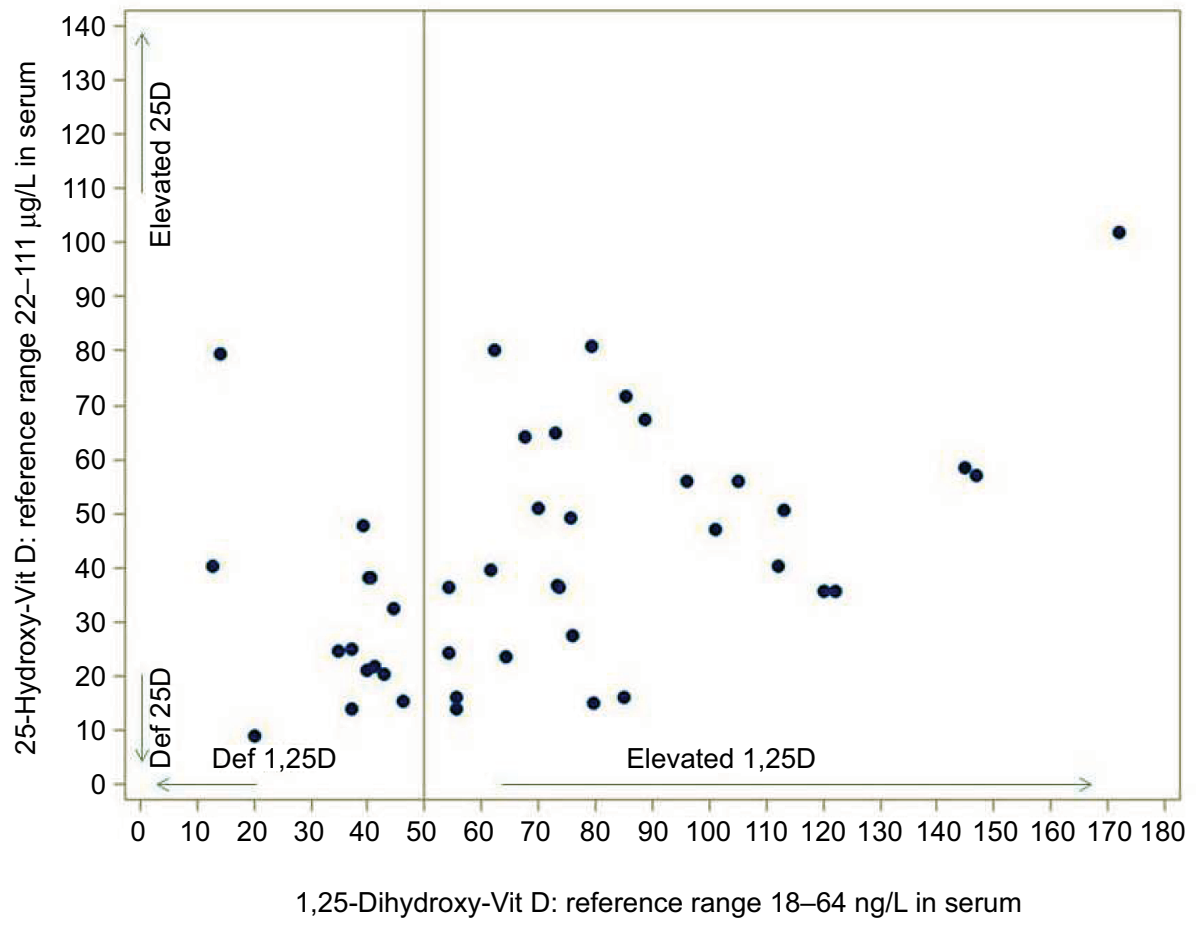

Figure 2 Distribution of $1,25 D$ and $25 D$ in the total study cohort $(n=43)$.

Abbreviations: I,25D, I,25-dihydroxyvitamin D3; Def, deficiency; 25D, 25-hydroxyvitamin D3.

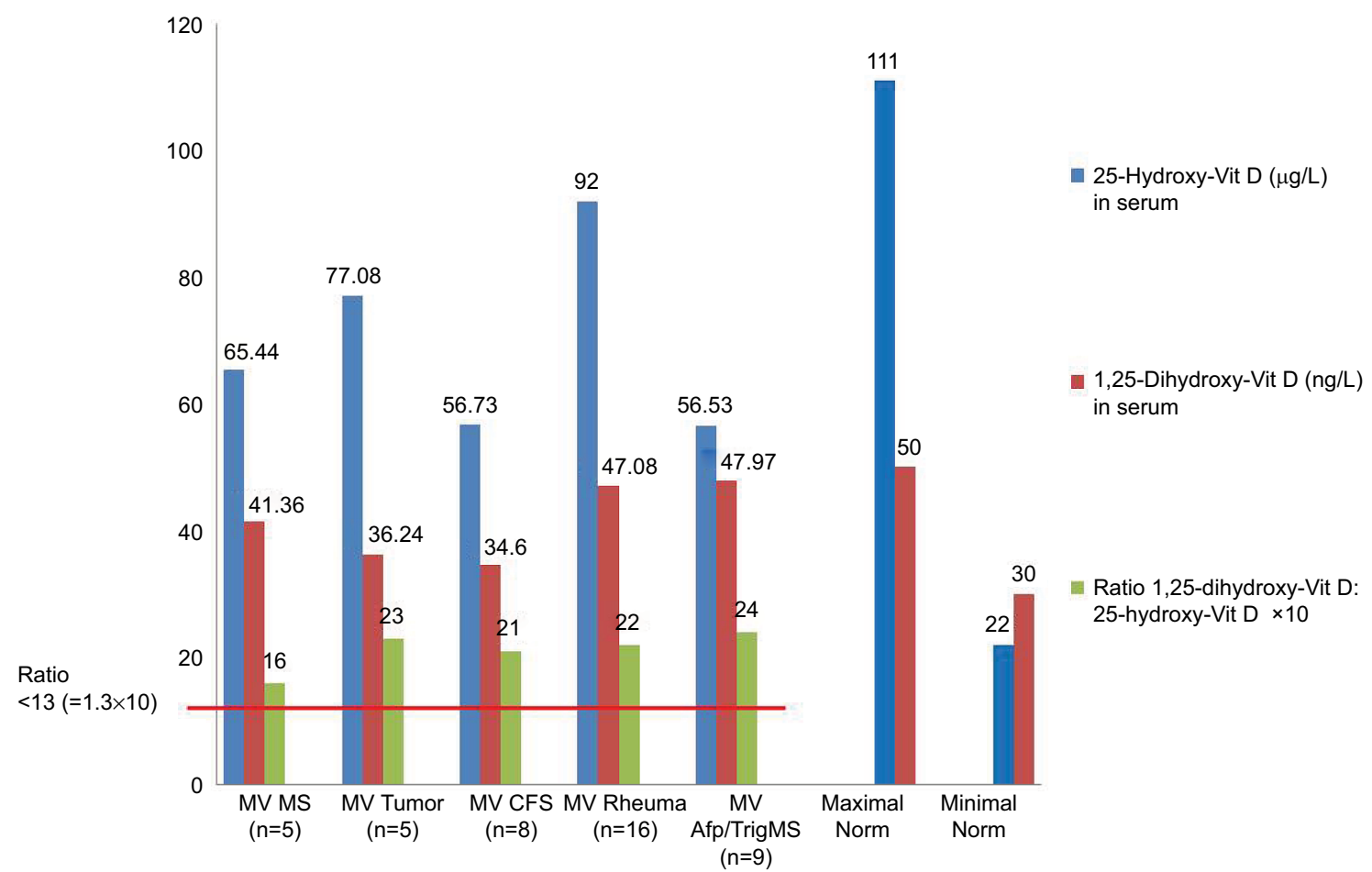

Figure 3 In all five disease groups, the 25D value is above the minimum threshold while the I,25D value falls within the standard range. (Vitamin D values are shown as mean values.) The distribution of the VDR ratio is greater than the assumed maximum of 1.3 ( $\times 10$ in the graph) in all five disease groups.

Abbreviations: I,25D, I,25-dihydroxyvitamin D3; 25D, 25-hydroxyvitamin D3; VDR, vitamin D receptor; MV, medium values; MS, multiple sclerosis; CFS, chronic fatigue syndrome; Afp/TrigMS, atypical facial pain/trigeminal myofacial symptoms; Rheuma, rheumatic; norm, normal.

ratio was $>1.3$. Based on the Kruskal-Wallis test $(P=0.6805)$, it may be assumed that the main tendencies of the disease groups do not differ. All the individual groups as well as the cohort as a whole had values $>1.3$. This shows that, from the cohort, VDR-deac was present in 34 patients with ISDs and clinically verified FDOJ. 


\section{Multiplex assay analysis of FDOJ}

The multiplex assay analysis of the control group of 19 healthy jawbone samples indicated the following three cytokines (in $\mathrm{pg} / \mathrm{mL}$ ): IL-1ra, 195.5 (SD \pm 569 ); FGF-2, 27.6 ( $\mathrm{SD} \pm 59$ ); and $\mathrm{R} / \mathrm{C}, 149.9$ ( $\mathrm{SD} \pm 127$ ). There were no corresponding values found in the literature for these mediators in healthy jawbone. The mean values obtained for the FDOJ cohort were noticeably higher than for the group of healthy jawbone samples (in pg/mL): FGF-2, 622.8; IL-1 ra, 616.6; and R/C, 4,590.8 ( $\mathrm{SD} \pm 2,536.35$ ). In Figure 4, the hyperactivated signal transduction of $\mathrm{R} / \mathrm{C}$ in the 43 FDOJ samples of the cohort is shown (red bars) in comparison to the 19 healthy jawbone samples (blue bars). Of particular note, IL-6, IL- 8 and TNF- $\alpha$ have extremely low values. These cytokines are regarded as the "ignitors of humoral defense", and their absence may explain the cryptic and asymptomatic character of FDOJ.

The cytokine profiles obtained from the FDOJ areas of all five groups demonstrate a common pattern (Figure 5): on one hand, there is a total paralysis of the immune system; on the other, there is a one-sided derailment of FGF-2, IL-1ra and $\mathrm{MCP}-1$, and, in particular, the extreme overexpression of $\mathrm{R} / \mathrm{C}$ in all five ISD groups.

\section{RANTES/CCL5 expression and the vitamin $D$ ratio}

Based on the aims of this study, the correlation (value $=0.13$ ) was calculated between $\mathrm{R} / \mathrm{C}$ expression in the FDOJ samples and VDR-deac. Using Spearman's correlation, it was found that this relationship was not significant $(P=0.39)$. The scatter plot diagram between $\mathrm{R} / \mathrm{C}$ and VDR-deac is shown in Figure 6. (See the "Limitations" section for various possible causes.)

\section{Discussion}

The discussion of the study findings presented addresses four factors concerning the possible etiological contribution of ISDs: the serum content of $1,25 \mathrm{D}(\mathrm{ng} / \mathrm{L})$; the serum content of $25 \mathrm{D}(\mu \mathrm{g} / \mathrm{L})$; the resulting calculated ratio; and the overexpression of $\mathrm{R} / \mathrm{C}$ (in $\mathrm{pg} / \mathrm{mL}$ ) in FDOJ. As noted previously, microbes - including Mycobacterium tuberculosis, Borrelia and Epstein-Barr virus (EBV) - downregulate VDR activity. In the event, thereby, that the physiologically active $1,25 \mathrm{D}$ form exceeds normal values, this may result in a corresponding reduction of 25D. Consequently, more vitamin D experts are beginning to reconsider vitamin D supplementation among the general population. ${ }^{25}$

\section{Serum levels of I,25D and 25D in the FDOJ cohort}

Despite the recent surge in vitamin D supplementation, the number of cases of chronic diseases has increased and is expected to continue to rise. ${ }^{26}$ Interpretation of the results presented in Figure 3 provides further supporting evidence with respect to this phenomenon. The FDOJ-ISD cohort exhibit the following relationships: the mean value of $1,25 \mathrm{D}$

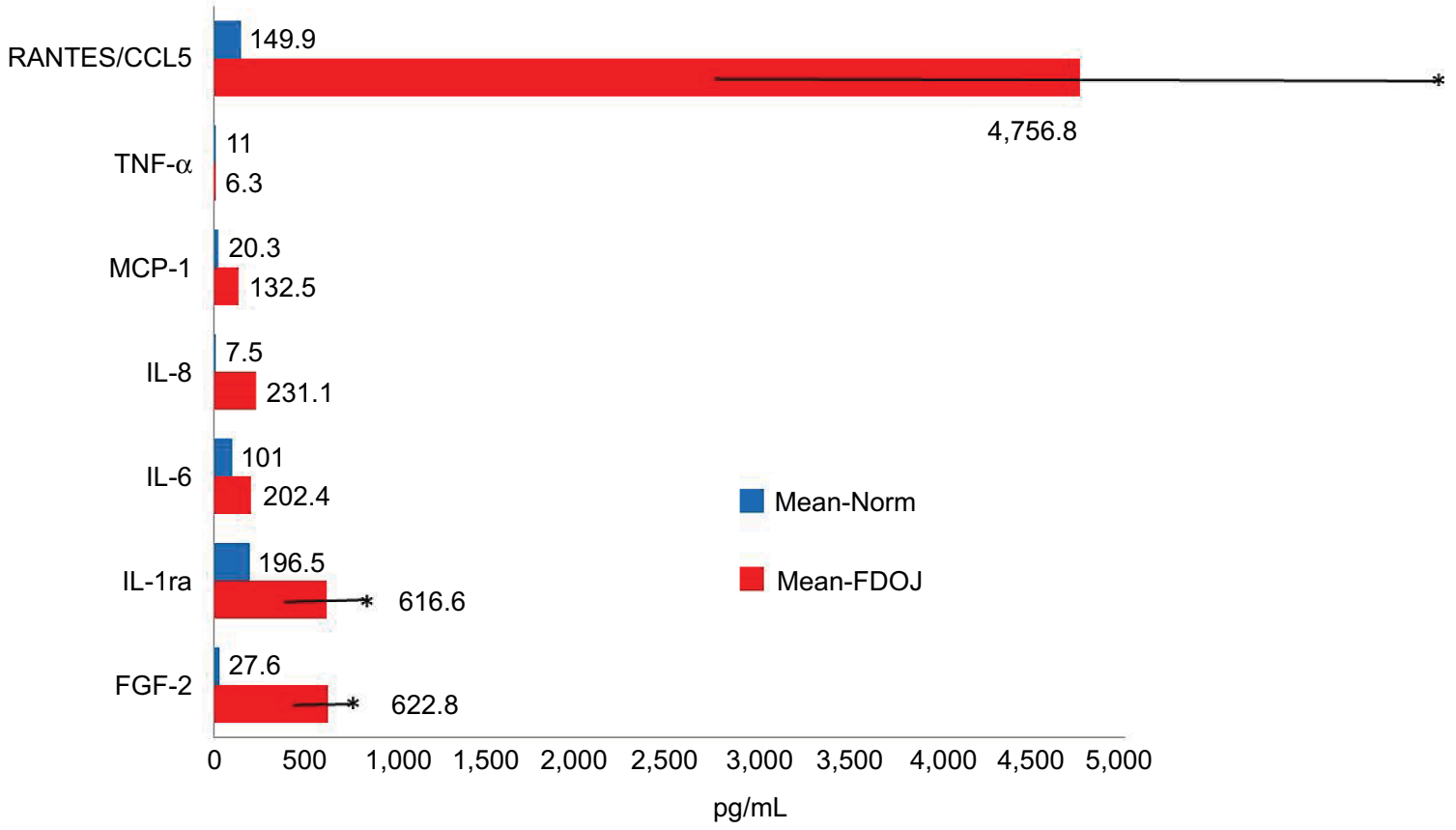

Figure 4 Comparison of the levels of seven cytokines obtained from 19 healthy jawbone samples with those from FDOJ samples from 43 patients with ISDs. Abbreviations: FDOJ, fatty-degenerative osteolysis of the jaw; ISD, immune system disease; TNF- $\alpha$, tumor necrosis factor alpha; MCP-I, monocyte chemotactic protein-I; IL, interleukin; IL-I ra, IL-I receptor antagonist; FGF-2, fibroblast growth factor-2; Norm, normal. 


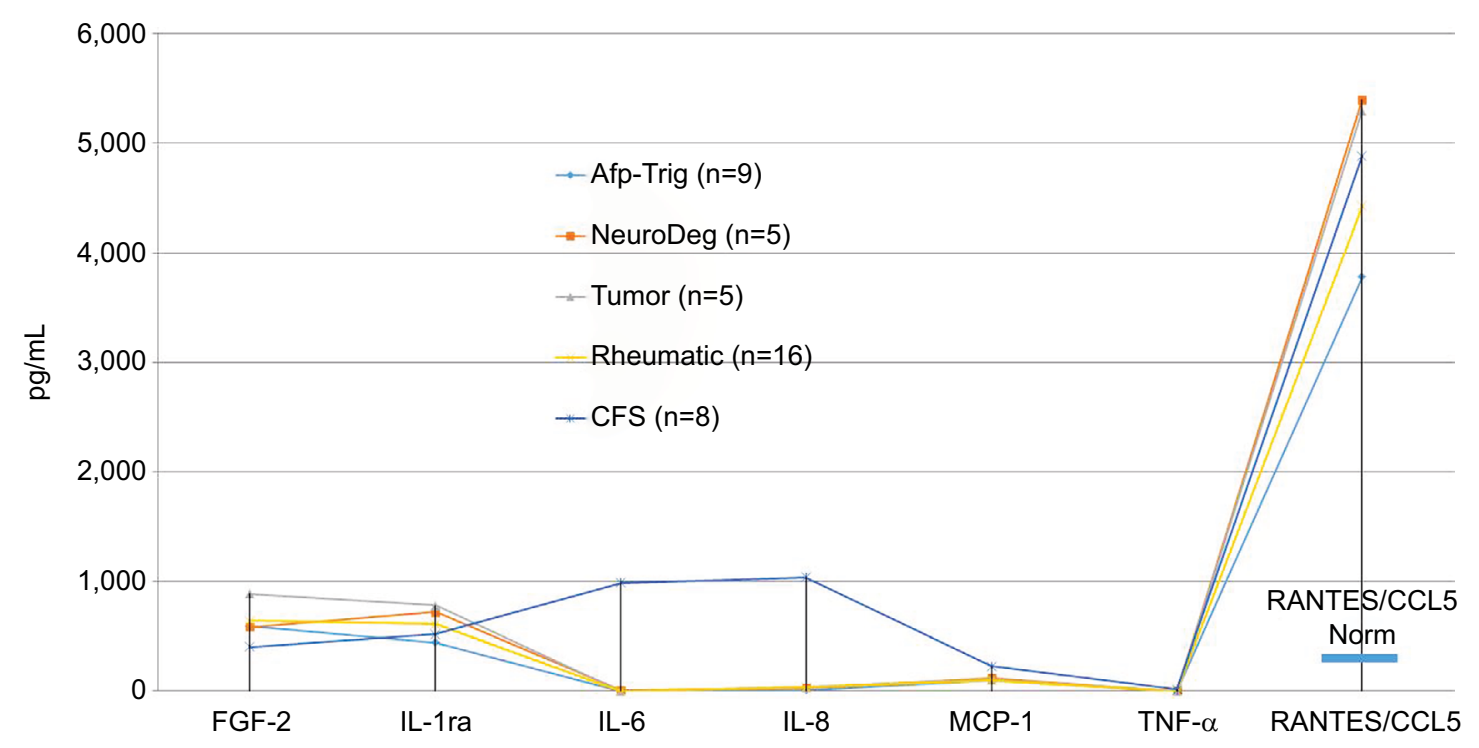

Figure 5 Distribution of seven cytokines in the FDOJ samples obtained from five disease groups: atypical facial and trigeminal pain ( $\mathrm{n}=9$ ); neurodegenerative disorders (multiple sclerosis and amyotrophic lateral sclerosis) ( $n=5$ ); tumors (breast cancer and prostate cancer) ( $n=5$ ); rheumatism (fibromyalgia and Lyme disease) ( $n=8$ ); and chronic fatigue syndrome $(n=8)$. There was significant overexpression of $R / C$ in all groups; there were no statistically significant differences between the individual groups (Kruskal-Wallis).

Abbreviations: FDOJ, fatty-degenerative osteolysis of the jaw; R/C, RANTES/CCL5; TNF- $\alpha$, tumor necrosis factor alpha; MCP-I, monocyte chemotactic protein-I; IL, interleukin; IL-I ra, IL-I receptor antagonist; FGF-2, fibroblast growth factor-2; Norm, normal; CFS, chronic fatigue syndrome; Afp-Trig, atypical facial pain-trigeminal Neuralgia; NeuroDeg, neurogenerative diseases.

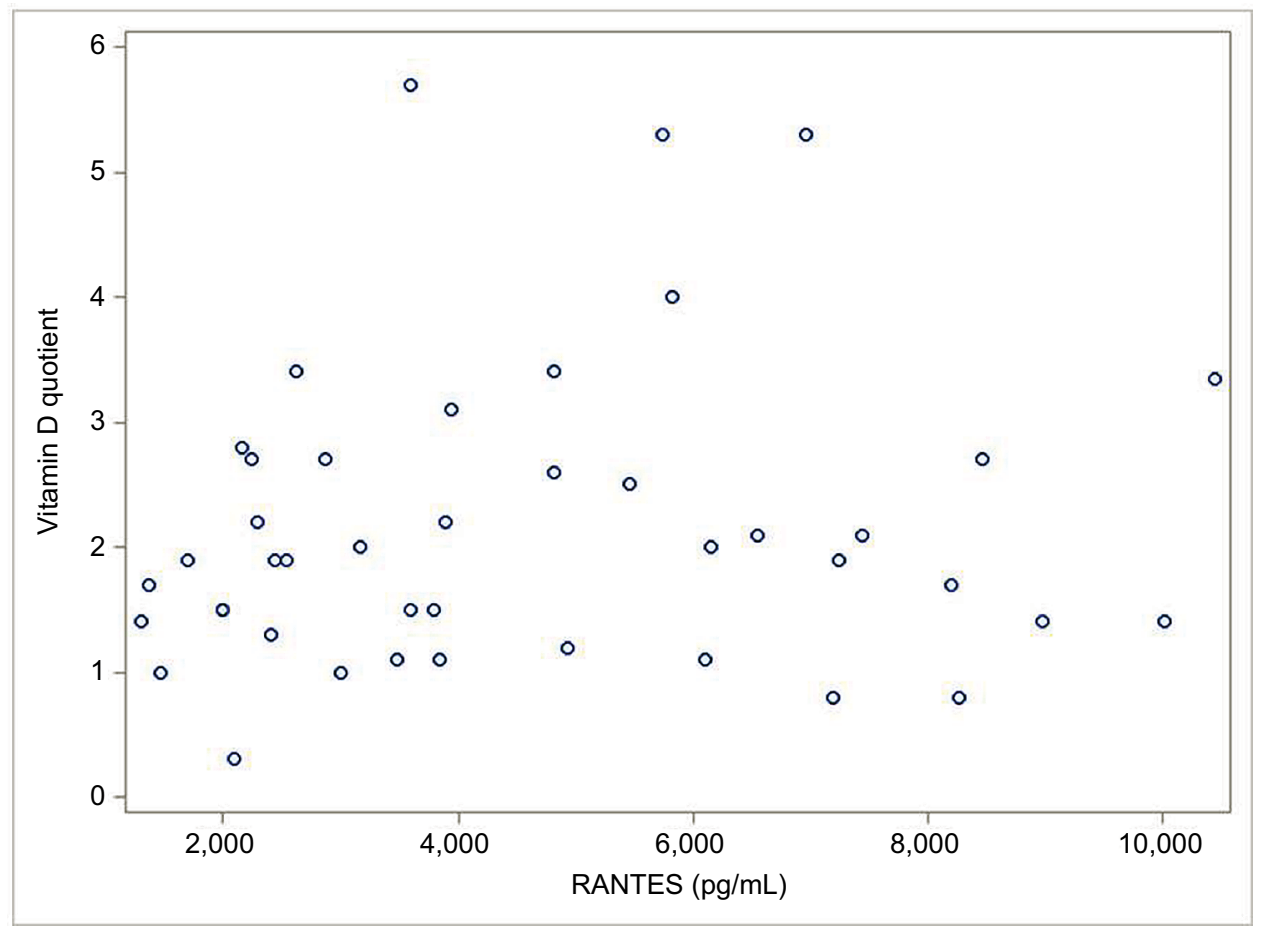

Figure 6 Correlation (value/r=0.13) between R/C expression in the FDOJ samples and the VDR-deac levels. Spearman's correlation coefficient is not significant ( $P=0.39$ ). Abbreviations: R/C, RANTES/CCL5; FDOJ, fatty-degenerative osteolysis of the jaw; VDR-deac, VDR deactivation.

of $71.11 \mathrm{ng} / \mathrm{L}$ ( $\mathrm{SD} \pm 36.0$ ) exceeds the maximum normal reference value of $50 \mathrm{ng} / \mathrm{L}$. At the same time, the mean value of $25 \mathrm{D}$ of $41.4 \mu \mathrm{g} / \mathrm{L}(\mathrm{SD} \pm 36.0$ ) falls in the lower part of the reference range of 111-22 $\mu \mathrm{g} / \mathrm{L}$ (Figure 3). Accordingly, an excess of the active hormone $1,25 \mathrm{D}$, in contrast to $25 \mathrm{D}$, is present. Autoimmune diseases often correlate with high $1,25 \mathrm{D}$ values and low $25 \mathrm{D}$ values (which is associated with a presumed "vitamin D deficiency"), and this is also the case in the FDOJ study cohort exclusively affected by ISDs: low 25D levels are inversely correlated with high 1,25D levels. 
Consistent with the disease patterns exhibited by the FDOJ cohort, abnormally low levels of the metabolite 25D are associated with general mortality and an increased incidence of at least 40 different chronic infections. ${ }^{27}$ Certainly, vitamin D intake is often purported to confer immunosuppressive effects. For instance, according to Arnson et al, "Vitamin D affects the immune system on many levels and via a number of mechanisms. Vitamin D has several immunosuppressive properties and, on the whole, has such an effect". ${ }^{28}$

\section{The phenomenon of deactivation of vitamin $D$ receptor}

The clinical evidence available for a wide range of autoimmune diseases shows that the innate immune function can be induced by restoring VDR function. ${ }^{29}$ Bacteria-induced ligands of the VDR deactivate this function. The infiltration of microorganisms into the cell is known to occur in the case of viruses. It is less known, however, whether bacteria can migrate into the cell by shape changing. The conversion of bacteria into so-called "cell wall-deficient forms" is a condition for their uptake within the cells. The bacteria that most effectively infiltrate the cells at the VDR are well-known agents of chronic infections (e.g., tuberculosis; borelliosis; Chlamydia infections; infections caused by all forms of herpes virus, EBV, cytomegaly virus; and Aspergillus sp. infections). Intracellular bacteria can modulate cytokine production $^{30}$ and in monocytes and macrophages cytokine activation markedly inhibits 1,25D/VDR gene transcription. Capnines are the active substances produced by these microbes and are capable of disabling the VDR. If VDR-deac is present, it is increasingly likely that the body will not attack its own tissues in autoimmune diseases; rather, antibodies are produced that are directed against certain parts of the metagenome communities of microbes. ${ }^{31}$ Intracellular microbes living in nuclear cells can interfere with DNA transcription and repair mechanisms, allowing them to trigger many of the dysfunctions associated with autoimmune diseases. Microbe immunosuppression succeeds as a result of VDR suppression. ${ }^{32}$ Defects in VDR signal transduction have previously been associated with bacterial infections and chronic inflammation. ${ }^{33}$ As early as 2010 , Proal et $\mathrm{al}^{31}$ reported that VDR influences at least 1,400 genes, many of which are associated with autoimmune disorders and cancer. ${ }^{34}$ In 2005 , Wang et $\mathrm{al}^{35}$ used in silico emulation to demonstrate that the sulfonolipid capnin, which is created by the biofilm bacterial species of the genera Cytophaga, Capnocytophaga, Sporocytophaga and Flexibacter, could bind to the VDR and thereby reduce its activity. ${ }^{33}$ Published models predict that as the increased concentrations of $1,25 \mathrm{D}$ accumulate in the nucleated cells, they will increasingly occupy the ligand-binding pockets of these receptors, thus displacing their endogenous ligands. ${ }^{36}$

\section{The connection between deactivated VDR and FDOJ: disrupted vitamin D metabolism and pathological morphology in the jawbone}

Mandibular bone remodeling is mediated by inflammatory factors such as cytokines and chemokines. Collectively, our data strongly point toward $\mathrm{R} / \mathrm{C}$ being an important molecule for communication between osteoclasts and osteoblasts, and shed new light on the functions of these chemokines in osteoblast biology. Vitamin D and its receptor metabolism also play an important role in bone resorption in immunemediated osteoporosis. In the case of FDOJ, previously documented by our team, ${ }^{14}$ questions arise concerning not only the effects but also the systemic causes of local areas of FDOJ. Our data show that there is no statistically significant correlation between FDOJ severity, expressed by the overexpression of R/C, and VDR-deac. Nevertheless, the presence of VDR-deac in 34 FDOJ-ISD patients (79\% of the study cohort), in addition to insufficient wound healing and chronic cytokine stimulation by root-filled teeth, ${ }^{37,38}$ may provide further answers with respect to the disorders of jawbone metabolism that arise in FDOJ. The aforementioned effects of VDR-deac may provide a further explanation for the chronic "silent inflammation" in the jaw, here referred to as FDOJ, that we have repeatedly reported..$^{14,18,37}$ At the same time, the chemokine $\mathrm{R} / \mathrm{C}$ is partly responsible for many ISDs - rheumatism, breast cancer, Hashimoto's thyroiditis, melanomas, multiple sclerosis, Amyotrophyic lateral sclerosis and so on; it serves as a key pathogenic element, being overexpressed by up to 35-fold in the affected jaw area.

We consider, therefore, the research findings of VDR deactivation in the development of FDOJ to be illuminating. VDR-deac also alters the balance of vitamin D-controlled metabolism in the jawbone which can lead to osteolysis of the trabecular structures and to the fatty transformation of medullar spaces. Morphologically, FDOJ thus presents as fatty clumps (Figures 1 and 7). The medullary bone lesion evident in FDOJ is microscopically similar to aseptic, ischemic osteonecrosis. ${ }^{17}$ FDOJ appears to represent the transition between the acute inflammation of a surgical dental wound and a chronically inflamed, disturbed area of the jaw.

Bisphosphonate-related osteonecrosis of the jaw (BRONJ) has been increasingly suspected as a potential complication of bisphosphonate therapy, and the controversy 


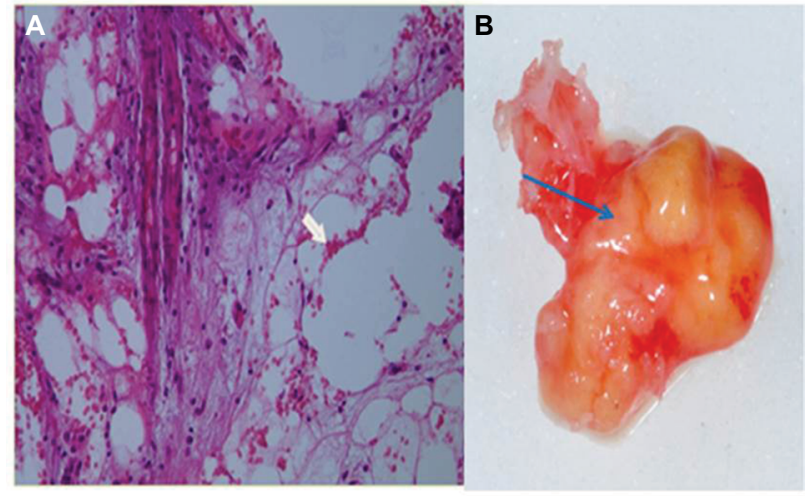

Figure 7 (A) A cluster of dead fatty cells (white arrow) with small inflammatory cells observed in the medullary cavity of the jaw. (B) An FDOJ tissue sample with complete fatty transformation of the cancellous portion of the jawbone (blue arrow). Abbreviation: FDOJ, fatty-degenerative osteolysis of the jaw.

with regard to the association between osteonecrosis of the jaw and bisphosphonates is a recent and growing problem. While FDOJ is normally painless and without visible bone loss, BRONJ is characterized by bone death wherein bone is destroyed in the course of turnover. ${ }^{41}$ In contrast, the upregulation of $\mathrm{R} / \mathrm{C}$ secretion from osteoblasts in response to the metabolism of FDOJ promotes osteoclast migration, but may also induce migration of additional preosteoblasts to the resorption site via a paracrine mechanism..$^{42}$ In this way, the process of bone modeling in the case of FDOJ is derailed but not connected to bone loss.

\section{Systemic signal transduction by hyperactivated RANTES/CCL5 from FDOJ areas}

At the cellular level, chronic inflammation is characterized by the infiltration of immunoinflammatory cells into the target tissue which usually precedes tissue damage. In areas of chronic inflammation, the production of cytokines by infiltrating and local tissue cells overwhelms and exceeds the regulatory mechanisms resulting in direct or indirect tissue destruction via the activation of immune and inflammatory cells. An imbalance between cytokines and their respective inhibitors is characteristic of chronic inflammatory states. Cytokines are involved in the induction of acute inflammatory events and later in the transition or persistence of chronic inflammation. This means that cytokine-producing mechanisms must be controlled in order to maintain healthy conditions. ${ }^{39}$ FDOJ exhibits a metabolic derailment and an associated chronic inflammatory input in the affected jaw region, which overexpresses $\mathrm{R} / \mathrm{C}$ by up to 35 times. This compromises the maintenance of regular cell signaling, thus causing persistent immune-mediated, metabolic and hemodynamic disturbances. These chronic stimuli can progress into diseases associated with local inflammation such as rheumatism, neurodegenerative diseases, tumors, chronic fatigue syndrome and facial pain (Figures 4 and 5). FDOJ is characterized by these metabolic and immunologic dysfunctions, with both adipocytes and macrophages being important cellular sensors and also effectors of metabolic derailments in bone metabolism. FDOJ constitutes its own unique inflammatory phenomenon, since the cell response is not bacterially or virally triggered but rather is initiated by persistent metabolic derailments. The entity responsible for these abacterial and aviral cell responses is primarily the proinflammatory chemokine $(\mathrm{R} / \mathrm{C})$. As a result, systemically relevant, dysregulated "signaling pathways" are activated as shown in the five groups of ISDs described in this study.

\section{Systemic cross-linking of VDR deactivation and osteolysis in the jawbone}

Mice lacking the cathelicidin gene, which is reliably transcribed by the VDR, exhibit longer wound-healing times than their wild types. ${ }^{33}$ This effect is regulated by the VDR only in humans and non-human primates, as the mouse cathelicidin gene does not possess a VDR binding site at the promoter. ${ }^{34}$ Thus, VDR-deac has a negative effect on wound healing. It may, therefore, be medically and systematically necessary to study the relationship between VDR-deac and bone metabolism in FDOJ. A previous study investigating the relationship between $\mathrm{R} / \mathrm{C}$ and 1,25D levels established yet a further connection: $\mathrm{R} / \mathrm{C}$ secretion from osteoblasts was inhibited by hormones such as $25 \mathrm{D}$ and dexamethasone. ${ }^{42}$ This means that if the effect of $1,25 \mathrm{D}$ is switched off intracellularly (i.e., if VDR-deac is present), the $\mathrm{R} / \mathrm{C}$ level would increase with a potentially negative immunomodulating effect on ISD. High 1,25D values promote osteoclast activity. ${ }^{43}$ The fact that excess $1,25 \mathrm{D}$ contributes to the successive formation of FDOJ corresponds with the observation that FDOJ and "vitamin D deficiency" are related. The research has shown that in response to $1,25 \mathrm{D}$, normal osteoclasts increase their production of acid hydrolases and subsequently increase their cell count. This means that osteoclast-mediated bone resorption is increased as a function of $1,25 \mathrm{D}$. High $1,25 \mathrm{D}$ values, in turn, are the result of chronic inflammation in conjunction with VDR-deac. Therefore, it is the chronic inflammatory process itself that causes osteoporosis and not "vitamin D deficiency". Low 25D values are only a consequence of the aforementioned contexts. Attempts to promote healing in 
FDOJ cases with excessive vitamin D supplementation are thus immunologically counterproductive. From the interrelations cited in the present study, it may be concluded that VDRdeac is a possible cause of the severe osteolysis observed in 34 of the 43 patients with FDOJ and of the extreme R/C overexpression. An almost complete absence of "ignitors of the immune system" (TNF- $\alpha$ and IL-6) in FDOJ areas (Figure 4) is another reason for the ailing defense present in those regions. Simultaneously, the VDR-deac prevents $1,25 \mathrm{D}$ from contributing to the expression of antimicrobial peptides (AMPs), such as cathelicidin and beta-defensin, which help to eliminate pathogens. ${ }^{44,45}$ In general, the innate immune system is stimulated by $1,25 \mathrm{D}$, and the adaptive immune system is inhibited. ${ }^{46,47}$ Thus, an effective immune response is highly dependent on the vitamin D3 endocrine system responsible for the balance of proinflammatory and anti-inflammatory elements. This relationship is illustrated by Blaney et $\mathrm{al}^{48}$ in a study of 100 patients with autoimmune and chronic diseases: $85 \%$ of patients showed $1,25 \mathrm{D}$ levels that were $>46.2 \mathrm{ng} / \mathrm{L}$ without hypercalcemia. Furthermore, large and reliable studies conducted with Danish population data found that the mean $1,25 \mathrm{D}$ value in a normal population was $29 \mathrm{ng} / \mathrm{L}$ with a standard deviation of 9.5. In addition, a review of the literature ${ }^{49}$ confirmed the association of elevated 1,25D levels with bone metabolism. It has been found that elevated $1,25 \mathrm{D}$ regulates VDR activity in the small intestine. This, in turn, transcribes and multiplies the genes that transport calcium and phosphorous across the intestinal epithelium. The mucosal response, and calcium and phosphorus resorption, is thus dependent on a competently activated VDR, while increased $1,25 \mathrm{D}$ reduces VDR competence. The fact that calcium and phosphorous resorption may be inhibited when VDR activity is impaired by increased $1,25 \mathrm{D}$ is illustrated by a study of Crohn's disease patients with elevated 1,25D levels and low bone mineral density. ${ }^{50}$ It was concluded that treating the underlying chronic inflammation improved the metabolic bone disease. Brot et $\mathrm{al}^{51}$ found that elevated $1,25 \mathrm{D}$ levels were associated with markedly reduced bone density and bone content as well as increased bone turnover. At levels $>42 \mathrm{ng} / \mathrm{L}, 1,25 \mathrm{D}$ stimulates osteoclast activity in the bone. This leads to osteoporosis development, tooth fractures and soft tissue calcification. ${ }^{52}$ Accordingly, it was found that a combination of high 1,25D and low 25D levels is associated with the lowest bone mineral levels and poorest bone health. ${ }^{50}$ The schematic overview in Figure 8 illustrates the interconnection between VDR-deac, autoimmune and systemic diseases, and FDOJ.

According to the existing theories on VDR-deac, our data indicate that both systemic problems and local FDOJ may be related to VDR-deac. Clinically, there may be an amplification loop for both factors: VDR-deac may trigger the development of FDOJ and ISDs; subsequently, FDOJ - which arises as a result of VDR-deac - also leads to ISD development via chronic $\mathrm{R} / \mathrm{C}$ overexpression. The discussion concerning the close relationship between bone metabolism, bone cells and the immune system opens up a new interdisciplinary research

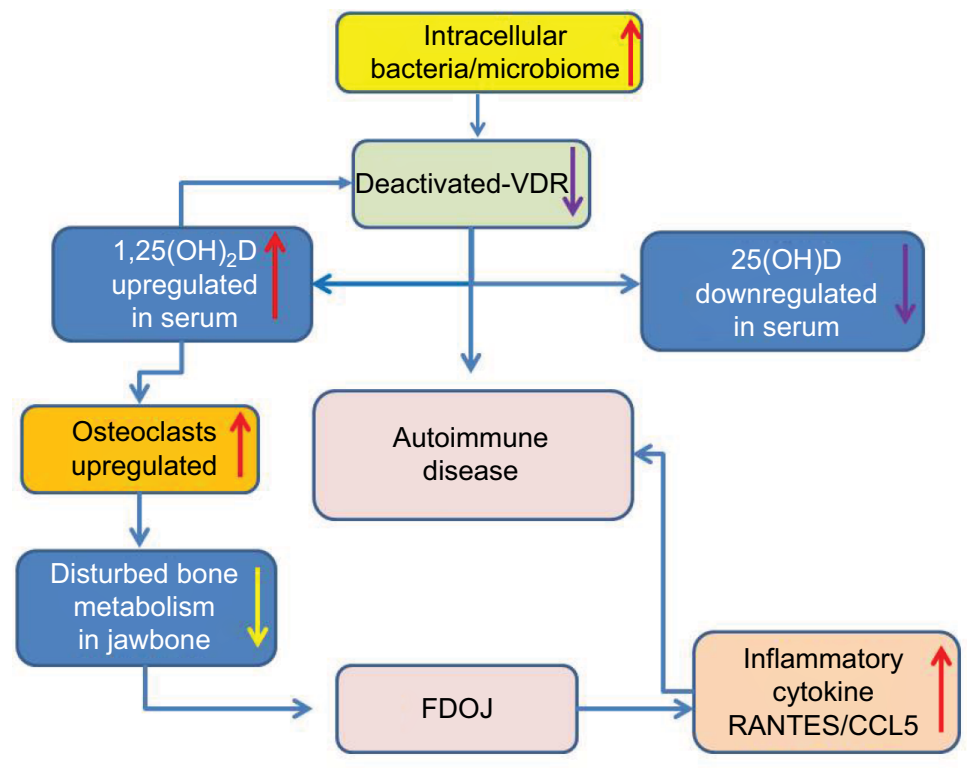

Figure 8 Interconnection between deactivated VDR, autoimmune and systemic diseases, disturbed bone metabolism and fatty-degenerative osteolysis of the jawbone with an immunological amplification loop.

Abbreviations: FDOJ, fatty-degenerative osteolysis of the jaw; VDR, vitamin D receptor; I,25(OH) 2 , I,25-dihydroxyvitamin D3; 25(OH)D, 25 -hydroxyvitamin D3. 
field known as "osteoimmunology". ${ }^{53}$ The data presented here highlight the complexity of interwoven pathways and the shared mechanisms involved in the cross talk between the immune and bone systems. Emerging new homeostatic networks define an interdisciplinary field of osteoimmunology in which other organs and systems are functionally interconnected. ${ }^{54}$ The conjunction of $\mathrm{R} / \mathrm{C}$ overexpression in FDOJ and the role of microbiota contributes to a proinflammatory cytokine milieu which drives bone resorption, leading to changes in bone density and in bone phenotype. ${ }^{55}$ Based on the overexpression of the chemokine $\mathrm{R} / \mathrm{C}$ in the FDOJ cases presented here, further studies investigating maxilla-mandibular osteoimmunology are recommended.

\section{Conclusion}

During the course of evolution, reactions to microbes have resulted in finely tuned immune responses. ${ }^{56}$ The present study brings the discussion on the subjects of the microbiome, as well as intracellular infections, and dysregulations of bone metabolism and signaling pathways into focus. We detail the interconnection between deactivated VDR, autoimmune and systemic diseases, disturbed bone metabolism and FDOJ osteolysis. In doing so, attention is drawn to the question of whether systemic cross-linking of VDR-deac and FDOJ-derived R/C overexpression may be responsible for the development of otherwise inexplicable systemic inflammatory reactions. We found that VDR-deac and metabolic regulation in the mineral matrix of the jawbone, combined with systemic dysregulated signal transduction, play a critical role in the development of the inflammatory condition FDOJ.

\section{Limitations}

The limitations of the cross-sectional study presented herein, in which vitamin D3 levels, cytokine levels and FDOJ samples were collected concurrently, lie in the small sample size employed. Futhermore, confounders were not controlled. These include the duration of any underlying disease, the duration of FDOJ, multimorbidity, medication use and the administration of other therapies. Bias may also be present in the determination of the VDR ratio. For instance, values were collected only once for each participant and at different seasonal times, which is limiting given that vitamin D levels are subject to natural fluctuations throughout the year.

\section{Future directions}

In a cohort of 43 patients with FDOJ, on the one hand, and ISDs, on the other, this study was able to demonstrate two phenomena that present similar effects to those associated with ISDs: aseptic osteolysis in the jawbone and the VDRdeac. Although the evidence for causality presented remains insufficient, our findings contribute to the expansion of the current understanding of chronic aseptic osteolysis of the jawbone, while also relating it to the immune system.

\section{Acknowledgment}

English-language translation and editing of this manuscript were done by Natasha Gabriel.

\section{Disclosure}

The authors report no conflicts of interest in this work.

\section{References}

1. Woolard MD, Frelinger JA. Outsmarting the host: bacteria modulating the immune response. Immunol Res. 2008;41(3):188-202.

2. Dermine JF, Desjardins M. Survival of intracellular pathogens within macrophages. Protoplasma. 1999;210(1-2):11-24.

3. Proal AD, Albert PJ, Blaney GP, LindsethIA, Benediktsson C, Marshall TG. Immunostimulation in the era of the metagenome. Cell Mol Immunol. 2011;8:213-225.

4. Liu PT., Stenger S, Li H, et al. Toll-like receptor triggering of a vitamin D-mediated human antimicrobial response. Science. 2006;311(5768):1770-1773.

5. WenL, Wong FS. How can the innate immune system influence autoimmunity in type 1 diabetes and other autoimmune disorders? Crit Rev Immunol. 2005. 25(3):225-250.

6. Fredricks DN, Reiman DA. Infectious agents and the etiology of chronic idiopathic diseases. Curr Clin Top Infect Dis. 1998;18:180-200.

7. Pordeus V, Szyper-Kravitz M, Levy RA, Vaz NM, Shoenfeld Y. Infections and autoimmunity: a panorama. Clin Rev Allergy Immunol. 2008;34(3): 283-299.

8. Shoenfeld Y, Isenberg DA. The mosaic of autoimmunity. Immunol Today. 10(4): 123-126.

9. Waterhouse JC, Perez TH, Albert PJ. Reversing bacteria-induced vitamin D receptor dysfunction is key to autoimmune disease. Ann $N$ Y Acad Sci. 2009;1173:757-765.

10. White JH. Vitamin D signaling, infectious diseases, and regulation of innate immunity. Infect Immun. 2008;76(9):3837-3843.

11. Hayes C, Nashold F, Spach K, Pedersen L. The immunological functions of the vitamin D endocrine system. Cell Mol Biol (Noisy-le-grand). 2003;49(2):277-300.

12. Topilski I, Flaishon L, Naveh Y, Harmelin A, Levo Y, Shachar I. The anti-inflammatory effects of 1,25-dihydroxyvitamin D3 on Th2 cells in vivo are due in part to the control of integrin-mediated T lymphocyte homing. Eur J Immunol. 2004;34(4):1068-1076.

13. Mangin M, Sinha R, Fincher K. Inflammation and vitamin D: the infection connection. InflammRes. 2014; 63(10):803-819.

14. Lechner J, von Baehr V. RANTES and fibroblast growth factor 2 in jawbone cavitations: triggers for systemic disease? Int J Gen Med. 2013;6: 277-290

15. Lau A, von Dossow V, Sander M, MacGuill M, Lanzke N, Spies C.. Alcohol use disorder and perioperative immune dysfunction. Anesth Analg. 2009;108(3):916-920.

16. Feltes BC, de Faria Poloni J, Nunes IJ, Bonatto D.. Fetal alcohol syndrome, chemo-biology and OMICS: ethanol effects on vitamin metabolism during neurodevelopment as measured by systems biology analysis. OMICS. 2014;18(6)344-363.

17. Ono K. Symposium: recent advances in avascular necrosis. Clin Orthop Rel Res.1992;277:2-138. 
18. Lechner J, Mayer W. Immune messengers in neuralgia inducing cavitational osteonecrosis (NICO) in jaw bone and systemic interference. Eur J Integr Med. 2010;2:71-77.

19. Ratner EJ, Langer B, Evins ML. Alveolar CAVITATional osteopathosis - manifestations of an infectious process and its implication in the causation of chronic pain. J Periodontol .1986;57(10):593-603.

20. Wang M, Jiao X, Meng Q, et al. Localization method in the diagnosis of the pathological jaw bone cavity. I Acta Acad Med Sichuan. 1982;13:341-344.

21. Marshall TG. VDR nuclear receptor is key to recovery from cognitive dysfunction. In: Days of molecular medicine, Abstract presentation; April 17-19; 2008; Karolinska Institute, Sweden.

22. Reinhardt TA, Horst RL. Self-induction of 1,25-dihydroxyvitamin D3 metabolism limits receptor occupancy and target tissue responsiveness. J Biol Chem. 1989;264(27):15917-15921.

23. Christakos S, Dhawan P, Verstuyf A, Verlinden L, Carmeliet G. Vitamin D: molecular mechanism of action, and pleiotrophic effects. Physiol Rev. 2016;96(1):365-408.

24. Calton EK, Keane KN, Newsholme P, Soares MJ. The impact of vitamin D levels on inflammatory status: a systematic review of immune cell studies. PLoS One. 2015;10(11):e0141770.

25. Tseng L. Controversies in vitamin D supplementation. eScholar- ship. 2003. Available from: http://www.escholarship.org/uc/item/4m84d4fn\# page-1. Accessed May 7, 2013.

26. Shelby J. Needs great, evidence lacking for people with multiple chronic conditions. Scribd. April 2013. Available from:https://chronicillnessrecovery.org/component/search/?searchword=Shelby\%20 J.\&searchphrase $=$ all\&Itemid=48. Accessed May 7, 2013.

27. Melamed ML, Michos ED, Post W, Astor B. 25-hydroxyvitamin D levels and the risk of mortality in the general population. Arch Intern Med. 2008;168:1629-1637.

28. Arnson Y, Amital H, Shoenfeld Y. Vitamin D and autoimmunity: new aetiological and therapeutic considerations. Ann Rheum Dis. 2007;66(9):1137-1142.

29. Arasaki K. Report on a case of systemic sarcoidosis treated according to the Marshall Protocol. In: The 26th Conference of the Japan Society of Sarcoidosis and Other Granulomatous Diseases. October 6; 2006; Tokyo, Japan.

30. Wang KX, Chen L. Helicobacter pylori L-form and patients with chronic gastritis. World J Gastroenterol. 2004;10(9): 1306-1309.

31. Proal AD, Albert PJ, Marshall TG. Autoimmune disease and the human metagenome. In: Nelson KE, editor. Metagenomics of the Human Body. New York: Springer; 2010: 231-275.

32. Sun J. Vitamin D and mucosal immune function. Curr Opin Gastroenterol. 2010;26(6): 591-595.

33. Auvynet C, Rosenstein Y. Multifunctional host defense peptides: antimicrobial peptides, the small yet big players in innate and adaptive immunity. FEBS J. 2009;276(22): 6497-6508.

34. Wei R, Christakos S. Mechanisms underlying the regulation of innate and adaptive immunity by vitamin D, Nutrients: 2015;7:8251-8260.

35. Wang TT, Tavera-Mendoza LE, Laperriere D, et al. Large-scale in silico and microarray-based identification of direct 1,25-dihydroxyvitamin $\mathrm{D}_{3}$ target genes. Mol Endocrinol. 2005;19(11):2685-2695.

36. Marshall TG. Vitamin D discovery outpaces FDA decision making. Bioessays. 2008;30(2): 173-182.

37. Proal AD, Albert PJ, Marshall TG. Dysregulation of the vitamin D nuclear receptor may contribute to the higher prevalence of some autoimmune diseases in women. Ann NY Acad Sci. 2009;1173: $252-259$.
38. Lechner J, von Baehr V. Chemokine R/C as an unknown link between wound healing in the jawbone and systemic disease: is prediction and tailored treatments in the horizon? EPMA J. 2015; 6:10.

39. Lechner J, von Baehr V. Stimulation of proinflammatory cytokines by volatile sulfur compounds in endodontically treated teeth. Int J Gen Med. 2015;10;9:109-118.

40. Kirkpatrick CJ, Fuchs S, Peters K, Brochhausen C, Hermanns MI, Unger RE. Visions for regenerative medicine: interface between scientific fact and science fiction. Artif Organs. 2006;30:822-827.

41. Gutta R, Louis PJ. Bisphosphonates and osteonecrosis of the jaws: science and rationale. Oral Surg Oral Med Oral Pathol Oral Radiol Endod. 2007;104(2):186-193.

42. Yano S. et al. Functional Expression of $\beta$-Chemokine receptors in osteoblasts: role of regulated upon activation, normal $\mathrm{T}$ cell expressed and secreted (RANTES) in osteoblasts and regulation of its secretion by osteoblasts and osteoclasts. Endocrinology. 2013;146(5):2324-2335.

43. Grise MA, Marks SC Jr, MacKay CA, Popoff SN. Effects of 1,25 dihydroxyvitamin $\mathrm{D}$ on osteoclast number and cytochemistry in normal and osteopetrotic (os) rabbits. Am J Anat.. 1990;189(3):261-266.

44. Lai Y, Gallo RL. AMPed up immunity: how antimicrobial peptides have multiple roles in immune defense. Trends Immunol. 2009;30(3):131-141.

45. Wang T, Nestel F, Bourdeau V, et al. Cutting edge: 1,25-di- hydroxyvitamin D3 is a direct inducer of antimicrobial peptide gene expression. J Immunol. 2004;173(5):2909-2912.

46. Hayes C, Nashold F, Spach K, Pedersen L. The immunological functions of the vitamin D endocrine system. Cell Mol Biol (Noisy-le-grand). 2003;49(2):277-300.

47. Topilski I, Flaishon L, Naveh Y, Harmelin A, Levo Y, Shachar I. The anti-inflammatory effects of 1,25-dihydroxyvitamin D3 on Th2 cells in vivo are due in part to the control of integrin-mediated T lymphocyte homing. Eur J Immunol. 2004;34(4):1068-1076.

48. Blaney GP, Albert PJ, Proal AD. Vitamin D metabolites as clinical markers in autoimmune and chronic disease. Ann NYAcad Sci. 2009;1173:384-390.

49. Abreu MT, Kantorovich V, Vasiliauskas EA, et al. Measurement of vitamin D levels in inflammatory bowel disease patients reveals a subset of Crohn's disease patients with elevated 1,25- dihydroxyvitamin D and low bone mineral density. Gut. 2004;53(8):1129-1136.

50. Ishizuka S, Kurihara N, Miura D, et al. Vitamin D antagonist, TEI-9647, inhibits osteoclast formation induced by 1alpha,25-dihydroxyvitamin D3 from pagetic bone marrow cells. J Steroid Biochem Mol Biol. 2004;89-90(1-5):331-334.

51. Brot C, Jørgensen N, Madsen OR, Jensen LB, Sørensen OH. Relationships between bone mineral density, serum vitamin $\mathrm{D}$ metabolites and calcium:phosphorus intake in healthy peri-menopausal women. J Intern Med. 1999;245(5):509-516.

52. Vanderschueren D, Pye SR, O'Neill TW, et al. Active vitamin D (1,25-dihydroxyvitamin D) and bone health in middle-aged and elderly men: the European Male Aging Study (EMAS). J Clin Endocrinol Metab. 2013;98(3):995-1005.

53. Horowitz M, Choi Y. Osteoimmunology: interactions of the bone and immune system. Endocr Rev. 2008;29(4):403-440.

54. Ginaldi L, De Martinis M. Osteoimmunology and beyond. Curr Med Chem. 2016;23(33):3754-3774

55. Hsu E, Pacifici R. From Osteoimmunology to osteomicrobiology: How the microbiota and the immune system regulate bone. Calcif Tissue Int 2017. Epub 2017 Oct 27.

56. Mangalam AK, Taneja V, David CS. HLA class II molecules influence susceptibility versus protection in inflammatory diseases by determining the cytokine profile. J Immunol. 2013;190(2):513-518. 


\section{Publish your work in this journal}

The International Journal of General Medicine is an international, peer-reviewed open-access journal that focuses on general and internal medicine, pathogenesis, epidemiology, diagnosis, monitoring and treatment protocols. The journal is characterized by the rapid reporting of reviews, original research and clinical studies across all disease areas.
The manuscript management system is completely online and includes a very quick and fair peer-review system, which is all easy to use. Visit http://www.dovepress.com/testimonials.php to read real quotes from published authors.

Submit your manuscript here: https://www.dovepress.com/international-journal-of-general-medicine-journal 